\title{
Data-Based Characteristics Analysis for Linear Discrete-Time Systems
}

\author{
Zhuo Wang, Derong Liu \\ The State Key Laboratory of Management and Control for Complex \\ Systems, Institute of Automation, Chinese Academy of Sciences, \\ Beijing 100190, China (e-mail: wangzhuo12300@gmail.com, \\ derong.liu@ia.ac.cn).
}

\begin{abstract}
In this paper, we develop data-based methods to analyze the characteristics of linear discrete-time systems, which have unknown parameter matrices. These characteristics include output controllability, asymptotic stability of the equilibrium point, bounded-input boundedstate stability, and bounded-input bounded-output stability. Our methods only use measured state and output data to verify the system properties. They are direct analysis methods and do not need to identify the unknown parameter matrices. These data-based methods not only can avoid identification errors, but also have lower computational complexity than traditional model-based analysis approaches.
\end{abstract}

Keywords: Data-based analysis; Linear discrete-time systems; Measured state and output data; System characteristics; Unknown parameter matrices.

\section{INTRODUCTION}

The modern industries have become considerably complex in manufacturing technologies, production processes and equipments. They have complicated characteristics, such as strong nonlinearities, uncertain operating system environments, strong coupling properties, as well as unknown dynamic parameters Chai et al. [2011]. As a consequence, engineers often met a lot of difficulties in the work of accurate model/parameter identifications Hjalmarsson et al. [2011], Rensfelt and Söderström [2011], which are established based on physical mechanisms using the first principle techniques. In this context, the traditional model-based methods, which are based on mathematical analysis and physical (chemical) mechanisms, have become more and more infeasible. People are seeking methodologies which do not rely on mathematical models, to analyze and control these kinds of systems.

Since information science and technology are widely used and are developing rapidly, we are in an era of big data. With the applications of the techniques such as digital sensors, digital storage, digital computation, and digital communication, etc., modern industries are generating huge amounts of online and offline data every day. Such data contain useful information and knowledge about the dynamical characteristics of the system. How to effectively utilize these data becomes a problem of great practical significance. Therefore, there is an urgent need to establish the data-based theories and the relevant methodologies to perform system modeling, analysis, control, decision making, and optimization.

When the mathematical model of the system is unknown, to analyze the system characteristics, traditional modelbased methods usually need to determine the structure of the system model and identify the corresponding pa- rameters first. Up until now, there are many methods for system identification and model construction, such as least squares methods Ding et al. [2011], Verhaegen and Verdult [2007], which can deal with noises and can avoid biased results; neural networks methods Lu [2011], Michalkiewicz [2012], which are good at nonlinear system identification; as well as support vector machine methods Liu et al. [2010], Zhang et al. [2009], which are performing well in regularization of system parameters, and are applicable to high-dimensional data sets. However, these model-based methods spend much time on system identification and cannot avoid the errors occurred during the identification process. According to this circumstance, data-based methods for system identification and model reduction are attracting attentions Battistelli et al. [2012], Rapisarda and Trentelman [2011], while direct system analysis methods using measured data, might also be a good choice.

Based on the above considerations, we will develop some data-based methods to analyze the characteristics of linear discrete-time systems. They are direct methods, which do not identify the unknown system parameter matrices. We will study the output controllability, the asymptotic stability of the equilibrium point, the input-to-state stability, and the input-to-output stability of the system. Our methods rely only on the measured data, and offer a new perspective on the study of system properties.

It must be pointed out that when the input takes effect, it is meaningful to study its influence on the system stability and whether the bounded input can result in bounded state and bounded output. This is because when there exists an input, if the state/output is unbounded, it may cause great damage or even collapse of the system, which will bring beyond recall loss. Therefore, we would like to study the bounded-input bounded-state (BIBS) and the bounded-input bounded-output (BIBO) stabilities. So far, 
there have been many researches published on this topic Jayawardhana et al. [2011], Sun et al. [2012], Moran and Labastida [2011].

\section{DATA-BASED METHODS FOR ANALYZING LINEAR DISCRETE-TIME SYSTEMS}

In this paper, we study linear discrete-time systems in the following form

$$
\left\{\begin{array}{l}
x(k+1)=A x(k)+B u(k) \\
y(k)=C x(k) \quad(k \geq 0)
\end{array}\right.
$$

where $x(k) \in R^{n}, u(k) \in R^{m}$, and $y(k) \in R^{q}$ are the state, the input, and the output of system (1), respectively. Matrices $A, B$, and $C$ are unknown, which do not have random variables as their elements. Assume that we can set the value of the initial state $x(0)$. In the following of this section, we will show that, one can directly verify the system properties only by using the measured state and output data, while does not need to identify the above matrices. We will also show that our data-based methods have lower computational complexity than the traditional model-based system analysis approaches.

\subsection{Data-Based Output Controllability Analysis}

In this subsection, we analyze the output controllability of system (1) using data-based methods.

Lemma 1. Mohandas [2006] System (1) is completely output controllable if and only if

$$
\operatorname{Rank}\left\{W_{O C}\right\}=\operatorname{Rank}\left\{\left[C B, C A B, \ldots, C A^{n-1} B\right]\right\}=q .
$$

In traditional model-based methods, to verify the output controllability of system (1), people need to identify $A, B$, and $C$, calculate $C A^{l} B(0 \leq l \leq n-1)$, and then check $\operatorname{Rank}\left\{W_{O C}\right\}$. The identification process usually introduces identification errors, and the calculation of $C A^{l} B$, which involves lots of multiplications and summations especially for high-dimensional systems, is quite complex.

In contrast, our data-based analysis method neither identifies the unknown matrices $A, B$, and $C$, nor calculates $C A^{l} B(0 \leq l \leq n-1)$. To begin with, we do $m$ groups of tests on system (1). Let all the state trajectories start from the same initial state $x^{[i]}(0)=0(1 \leq i \leq m)$, while setting the corresponding inputs as

$$
u^{[i]}(k) \equiv u^{[i]}=[0, \ldots, 0,1,0, \ldots, 0]^{T} \quad(1 \leq i \leq m),
$$

whose $i$ th element is 1 and all other elements are zeros. Therefore, $y^{[i]}(0)=0$. Measure and record $y^{[i]}(1), y^{[i]}(2)$, $\ldots, y^{[i]}(n), \ldots$ Define

$$
\begin{aligned}
& Z(k)=\left[y^{[1]}(k), y^{[2]}(k), \ldots, y^{[m]}(k)\right] \quad(0 \leq k \leq n), \\
& V_{j}=Z(j)-Z(j-1) \quad(1 \leq j \leq n), V=\left[V_{1}, V_{2}, \ldots, V_{n}\right],
\end{aligned}
$$

where $Z(k) \in R^{q \times m}$. Note that $Z(0)=0$. With the above preparations, we present the following theorem on the output controllability of system (1).

Theorem 1. System (1) is completely output controllable if and only if $\operatorname{Rank}\{V\}=q$, where $V$ is defined in (3).
Proof. Since $x^{[i]}(0)=0(1 \leq i \leq m)$, when the inputs $u^{[i]}(k)$ are set as in (2), the outputs of system (1) will be

$$
\left\{\begin{aligned}
y^{[i]}(1) & =C B u^{[i]} \\
y^{[i]}(2) & =C A B u^{[i]}+C B u^{[i]} \\
& \vdots \\
y^{[i]}(n) & =C A^{n-1} B u^{[i]}+\cdots+C A B u^{[i]}+C B u^{[i]} .
\end{aligned}\right.
$$

From (3) and (4), we shall have

$$
\left\{\begin{aligned}
Z(0) & =0 \\
Z(1) & =C B \\
Z(2) & =C A B+C B \\
& \vdots \\
Z(n) & =C A^{n-1} B+\cdots+C A B+C B .
\end{aligned}\right.
$$

Such that

$$
\begin{aligned}
& V_{j}=C A^{j-1} B \quad(1 \leq j \leq n) \\
& V=\left[C B, C A B, \ldots, C A^{n-1} B\right]=W_{O C} .
\end{aligned}
$$

By Lemma 1, system (1) is completely output controllable if and only if $\operatorname{Rank}\{V\}=q$.

Corollary 1. Suppose that $q=n$. System (1) is completely state controllable if $\operatorname{Rank}\{V\}=n$, where matrix $V$ is defined in (3).

Proof. Let $W_{C}=\left[B, A B, \ldots, A^{n-1} B\right]$, which is the state controllability matrix. The necessary and sufficient condition for system (1) to be completely state controllable is $\operatorname{Rank}\left\{W_{C}\right\}=n$. Since $q=n$ and $V=W_{O C}=C W_{C}$,

$$
\operatorname{Rank}\{V\} \leq \min \left\{\operatorname{Rank}\{C\}, \operatorname{Rank}\left\{W_{C}\right\}\right\} \leq n .
$$

If $\operatorname{Rank}\{V\}=n$, then $\operatorname{Rank}\left\{W_{C}\right\}=n$. But, the inverse may not be true because $\operatorname{Rank}\{C\} \leq n$.

Therefore, when $q=n$, the sufficient condition for system (1) to be completely state controllable is $\operatorname{Rank}\{V\}=n$.

\subsection{Data-Based Stability Analysis}

In this subsection, we will present some data-based methods to analyze the stabilities of system (1), which include the asymptotic stability of the equilibrium point, the input-to-state stability, and the input-to-output stability. Before that, we provide the following definitions.

Definition 1. Antsaklis and Michel [2006] If every bounded input of a system results in a bounded state, then that system is called bounded-input bounded-state (BIBS) stable. More specifically, system (1) is BIBS stable if there are two constants $0<L_{u}, L_{x}<\infty$ such that the conditions

$$
x(0)=0, \quad\|u(k)\| \leq L_{u}, \quad k \geq 0,
$$

(3) imply that $\|x(k)\| \leq L_{x}$ for all $k \geq 0$.

Definition 2. Antsaklis and Michel [2006] If every bounded input of a system results in a bounded output, then that system is called bounded-input bounded-output (BIBO) stable. More specifically, system (1) is BIBO stable if there are two constants $0<L_{u}, L_{y}<\infty$ such that the conditions

$$
x(0)=0, \quad\|u(k)\| \leq L_{u}, \quad k \geq 0,
$$


imply that $\|y(k)\| \leq L_{y}$ for all $k \geq 0$.

Recall the $m$ groups of tests on system (1) and measured data $y^{[i]}(1), y^{[i]}(2), \ldots, y^{[i]}(n), \ldots(1 \leq i \leq m)$ introduced in Subsection 2.1, we have the following theorem.

Theorem 2. If $\left\|y^{[i]}(\infty)\right\|=M_{i}<\infty(1 \leq i \leq m)$, then system (1) is BIBO stable.

Proof. By (1) and (2), with $x^{[i]}(0)=0$, we shall have

$$
y^{[i]}(k+1)=C \sum_{j=0}^{k} A^{k-j} B u^{[i]} \quad(1 \leq i \leq m) .
$$

Suppose that the different eigenvalues of $A$ are $\lambda_{1}, \ldots, \lambda_{s}$ $(1 \leq s \leq n)$, and the multiplicity of $\lambda_{h}(1 \leq h \leq s)$ is $m_{h}$. Then, $\sum_{h=1}^{s} m_{h}=n$. Assume that $J \in \bar{R}^{n \times n}$ is the Jordan canonical form of $A$. There exists a nonsingular matrix $P \in R^{n \times n}$, such that $A=P J P^{-1}, A^{j}=P J^{j} P^{-1}$ $(0 \leq j \leq k)$, where $J^{j}=\operatorname{diag}\left[J_{1}^{j}\left(\lambda_{1}\right), \ldots, J_{s}^{j}\left(\lambda_{s}\right)\right]$ and

$$
J_{h}^{j}\left(\lambda_{h}\right)=\left[\begin{array}{cccc}
\lambda_{h}^{j} \mathcal{C}_{j}^{1} \lambda_{h}^{j-1} & \cdots & \mathcal{C}_{j}^{m_{h}-1} \lambda_{h}^{j-m_{h}+1} \\
0 & \lambda_{h}^{j} & \ddots & \vdots \\
\vdots & \vdots & \ddots & \mathcal{C}_{j}^{1} \lambda_{h}^{j-1} \\
0 & 0 & \cdots & \lambda_{h}^{j}
\end{array}\right] .
$$

Any nonzero element of $J_{h}^{j}\left(\lambda_{h}\right)$ can be denoted by $\mathcal{C}_{j}^{l} \lambda_{h}^{j-l}$, where $\mathcal{C}_{j}^{l}=\frac{j !}{l !(j-l) !}\left(0 \leq l \leq m_{h}-1, l \leq j\right)$.

Let $H^{[i]}(k-j)=J^{k-j} P^{-1} B u^{[i]}$. Then,

$$
y^{[i]}(k+1)=C P \sum_{j=0}^{k} H^{[i]}(k-j) .
$$

Since $P^{-1} B u^{[i]}$ is a constant vector, each element of $H^{[i]}(k-j)$ is in the form of $\beta_{k-j-l}^{h} \lambda_{h}^{k-j-l}(0 \leq l \leq$ $m_{h}-1$ and $\left.l \leq k-j\right)$, where $\beta_{k-j-l}^{h}$ is a real number. Because $\left\|y^{[i]}(\infty)\right\|=M_{i}<\infty$, by Abel's Theorem, $\lim _{k \rightarrow \infty} \sum_{j=0}^{k} \beta_{k-j-l}^{h} \lambda_{h}^{k-j-l}$ absolutely converges, and $\lim _{k \rightarrow \infty} \sum_{j=0}^{k} H^{[i]}(k-j)$ absolutely converges too. This means that $\lim _{k \rightarrow \infty} \sum_{j=0}^{k} H_{p}^{[i]}(k-j) L_{u}$ absolutely converges, where $H_{p}^{[i]}(k-j)$ is the $p$ th $(1 \leq p \leq n)$ element of $H^{[i]}(k-j)$, and $\|u(k)\| \leq L_{u}<\infty$ for all $k \geq 0$.

Next, we study the case that the input is $u_{\alpha}^{[i]}(k)=\alpha_{i}(k) u^{[i]}$ $(1 \leq i \leq m)$, where $\alpha_{i}(k)$ is a real number. Thus, the output is

$$
y_{\alpha}^{[i]}(k+1)=C P \sum_{j=0}^{k} H^{[i]}(k-j) \alpha_{i}(j) .
$$

Let $\eta_{\alpha}^{[i]}(k+1)=\sum_{j=0}^{k} H^{[i]}(k-j) \alpha_{i}(j)$, so $y_{\alpha}^{[i]}(k+1)=$ $C P \eta_{\alpha}^{[i]}(k+1)$. Let $y_{\alpha, r}^{[i]}(k)$ and $\eta_{\alpha, p}^{[i]}(k)$ denote the $r$ th $(1 \leq r \leq q)$ and the $p$ th $(1 \leq p \leq n)$ elements of $y_{\alpha}^{[i]}(k)$ and $\eta_{\alpha}^{[i]}(k)$, respectively. Then, we have

$$
\eta_{\alpha, p}^{[i]}(k+1)=\sum_{j=0}^{k} H_{p}^{[i]}(k-j) \alpha_{i}(j) .
$$

Because $\lim _{k \rightarrow \infty} \sum_{j=0}^{k} H_{p}^{[i]}(k-j) L_{u}=\sum_{j=0}^{\infty} H_{p}^{[i]}(j) L_{u}$ absolutely converges,

$$
\sum_{j=0}^{\infty} H_{p}^{[i]}(j) L_{u}=\sum_{j_{1}=0}^{\infty} H_{p}^{[i]}\left(j_{1}\right) L_{u}-\sum_{j_{2}=0}^{\infty}\left|H_{p}^{[i]}\left(j_{2}\right)\right| L_{u}
$$

where $H_{p}^{[i]}\left(j_{1}\right) \geq 0$ and $H_{p}^{[i]}\left(j_{2}\right)<0 ; \sum_{j_{1}=0}^{\infty} H_{p}^{[i]}\left(j_{1}\right) L_{u}$ and $\sum_{j_{2}=0}^{\infty}\left|H_{p}^{[i]}\left(j_{2}\right)\right| L_{u}$ both converge. Because $\left|\alpha_{i}(k)\right| \leq L_{u}$,

$$
\begin{gathered}
\left|H_{p}^{[i]}\left(j_{1}\right) \alpha_{i}\left(j_{1}\right)\right| \leq H_{p}^{[i]}\left(j_{1}\right) L_{u}, \\
\left|H_{p}^{[i]}\left(j_{2}\right) \alpha_{i}\left(j_{2}\right)\right| \leq\left|H_{p}^{[i]}\left(j_{2}\right)\right| L_{u} .
\end{gathered}
$$

According to Weierstrass Theorem, $\sum_{j_{1}=0}^{\infty} H_{p}^{[i]}\left(j_{1}\right) \alpha_{i}\left(j_{1}\right)$ and $\sum_{j_{2}=0}^{\infty} H_{p}^{[i]}\left(j_{2}\right) \alpha_{i}\left(j_{2}\right)$ both converge. In summary, $\lim _{k \rightarrow \infty} \eta_{\alpha, p}^{[i]}(k+1)=\lim _{k \rightarrow \infty} \sum_{j=0}^{k} H_{p}^{[i]}(k-j) \alpha_{i}(j)$ converges. So, $\lim _{k \rightarrow \infty} \eta_{\alpha}^{[i]}(k)$ converges, i.e. $\lim _{k \rightarrow \infty} y_{\alpha}^{[i]}(k)=C P \lim _{k \rightarrow \infty} \eta_{\alpha}^{[i]}(k)$ converges. This indicates that any bounded input $u_{\alpha}^{[i]}(k)=$ $\alpha_{i}(k) u^{[i]}$ will result in a bounded output $y_{\alpha}^{[i]}(k)$.

More generally, any input $u(k)$ can be expressed as $u(k)=$ $\sum_{i=1}^{m} \alpha_{i}(k) u^{[i]}$. Without loss of generality, we let $x(0)=0$. Then, we can obtain

$$
\begin{aligned}
y(k+1) & =C \sum_{i=1}^{m} \sum_{j=0}^{k} A^{k-j} B u^{[i]} \alpha_{i}(j) \\
& =\sum_{i=1}^{m} y_{\alpha}^{[i]}(k+1) .
\end{aligned}
$$

Since $y_{\alpha}^{[i]}(k)(1 \leq i \leq m)$ are bounded with $\|u(k)\| \leq L_{u}$ $(k \geq 0)$, then any bounded $u(k)$ will result in bounded $y(k)$. By Definition 2, the system is BIBO stable.

Lemma 2. Huang [2003] If system (1) is completely state observable, the BIBS stability and the BIBO stability of the system are equivalent.

Lemma 2 implies that as long as system (1) is completely state observable, we can verify the BIBS stability using the data-based analysis method introduced in Theorem 2 .

To verify the state observability of system (1), the traditional model-based methods need to identify $A$ and $C$ first, and construct the observability matrix

$$
W_{O}=\left[\begin{array}{c}
C \\
C A \\
\vdots \\
C A^{n-1}
\end{array}\right],
$$

then check whether $\operatorname{Rank}\left\{W_{O}\right\}=n$.

In Wang and Liu [2011], they developed a data-based analysis method, which can directly verify the state observability without identifying $A$ and $C$. This data-based analysis method also starts with some tests and measurements. Set $n$ linearly independent nonzero initial states as

$$
\bar{x}^{[i]}(0)=[0, \ldots, 0,1,0, \ldots, 0]^{T} \quad(1 \leq i \leq n),
$$


where the $i$ th element of $\bar{x}^{[i]}(0)$ is 1 , and all other elements are zeros. Set the corresponding inputs as $\bar{u}^{[i]}(k) \equiv 0$. Measure and record the corresponding outputs $\bar{y}^{[i]}(0), \bar{y}^{[i]}(1), \ldots, \bar{y}^{[i]}(n-1)$.

Define

$$
\begin{aligned}
& \hat{Y}(k)=\left[\bar{y}^{[1]}(k), \bar{y}^{[2]}(k), \ldots, \bar{y}^{[n]}(k)\right] \quad(0 \leq k \leq n-1), \\
& Q=\left[\begin{array}{c}
\hat{Y}(0) \\
\hat{Y}(1) \\
\vdots \\
\hat{Y}(n-1)
\end{array}\right],
\end{aligned}
$$

where $\hat{Y}(k) \in R^{q \times n}$, and actually $Q=W_{O}$. We then have the following state observability criterion.

Lemma 3. Wang and Liu [2011] Assume that under no input condition, the initial states of system (1) can be set as in (9). Then, the system is completely state observable, if and only if $\operatorname{Rank}\{Q\}=n$, where $Q$ is defined in (10).

Theorem 3. For system (1), if

1) $\left\|y^{[j]}(\infty)\right\|=M_{j}<\infty(1 \leq j \leq m)$, where measured data $y^{[j]}(1), y^{[j]}(2), \ldots, y^{[j]}(n), \ldots$ are introduced in Subsection 2.1;

2) under no input condition, the initial states of system (1) can be set as in (9) and $\operatorname{Rank}\{Q\}=n$, where $Q$ is defined in (10);

then

1) the system is both BIBS and BIBO stable;

2 ) the equilibrium point $x=0$ is stable.

Proof. With Condition 1), by Theorem 2, system (1) is BIBO stable. According to Condition 2) and Lemmas 2 and 3 , the system is completely state observable, and thus it is also BIBS stable.

If we set $u(k) \equiv 0$, by Definition $1,\|u(k)\|<L_{u}$ and $\|x(k)\| \leq L_{x}$ for all $k \geq 0$, where $0<L_{u}, L_{x}<\infty$ are two constants. Then, the equilibrium point $x=0$ is stable.

Lemma 4. Antsaklis and Michel [2006] The following statements are equivalent:

- The equilibrium point $x=0$ is asymptotically stable.

- The equilibrium point $x=0$ is asymptotically stable in the large.

- $\lim _{k \rightarrow \infty}\left\|A^{k}\right\|=0$.

Theorem 4. Set $n$ linearly independent initial states $\bar{x}^{[1]}(0)$, $\bar{x}^{[2]}(0), \ldots, \bar{x}^{[n]}(0)$ as in $(9)$. Assume that $\operatorname{Rank}\{Q\}=n$, where $Q$ is defined in (10). Measure and record $\bar{x}^{[1]}(1)$, $\bar{x}^{[2]}(1), \ldots, \bar{x}^{[n]}(1)$. If

$$
\left\|\left[\bar{x}^{[1]}(1), \ldots, \bar{x}^{[n]}(1)\right]\left[\bar{x}^{[1]}(0), \ldots, \bar{x}^{[n]}(0)\right]^{-1}\right\|<1,
$$

then

(1) the equilibrium point $x=0$ is asymptotically stable (in the large);

(2) system (1) is both BIBS and BIBO stable.
Proof. By Lemma 3, because $\operatorname{Rank}\{Q\}=n$, the system is completely state observable. Therefore, we can measure and record the values of $\bar{x}^{[1]}(1), \bar{x}^{[2]}(1), \ldots, \bar{x}^{[n]}(1)$.

With $\bar{u}^{[i]}(k) \equiv 0(1 \leq i \leq n)$, we shall have

$$
\bar{x}^{[i]}(1)=A \bar{x}^{[i]}(0) \quad(1 \leq i \leq n) .
$$

Such that $\left[\bar{x}^{[1]}(1), \ldots, \bar{x}^{[n]}(1)\right]=A\left[\bar{x}^{[1]}(0), \ldots, \bar{x}^{[n]}(0)\right]$. Since $\bar{x}^{[1]}(0), \bar{x}^{[2]}(0), \ldots, \bar{x}^{[n]}(0)$ are linearly independent, then $\left[\bar{x}^{[1]}(0), \ldots, \bar{x}^{[n]}(0)\right]$ is invertible. As a consequence,

$$
\begin{aligned}
& A=\left[\bar{x}^{[1]}(1), \ldots, \bar{x}^{[n]}(1)\right]\left[\bar{x}^{[1]}(0), \ldots, \bar{x}^{[n]}(0)\right]^{-1}, \\
& \|A\|=\left\|\left[\bar{x}^{[1]}(1), \ldots, \bar{x}^{[n]}(1)\right]\left[\bar{x}^{[1]}(0), \ldots, \bar{x}^{[n]}(0)\right]^{-1}\right\| .
\end{aligned}
$$

Under the given condition, $\|A\|<1$. Thus,

$$
\lim _{k \rightarrow \infty}\|A\|^{k}=0 \text {. }
$$

Because $0 \leq\left\|A^{k}\right\| \leq\|A\|^{k}(k \geq 0)$, we can obtain

$$
\lim _{k \rightarrow \infty}\left\|A^{k}\right\|=0 \text {. }
$$

By Lemma 4 , the equilibrium point $x=0$ is asymptotically stable (in the large). For system (1), we have

$$
\begin{aligned}
& y(k+1)=C A^{k+1} x(0)+\sum_{j=0}^{k} C A^{j} B u(k-j) . \\
& \|y(k+1)\| \\
& =\left\|C A^{k+1} x(0)+\sum_{j=0}^{k} C A^{j} B u(k-j)\right\| \\
& \leq\|C\|\left[\|A\|^{k+1}\|x(0)\|+\sum_{j=0}^{k}\|A\|^{j}\|B\|\|u(k-j)\|\right] \\
& \leq\|C\|\left[\|A\|^{k+1}\|x(0)\|+\sum_{j=0}^{\infty}\|A\|^{j}\|B\|\|u(k-j)\|\right] .
\end{aligned}
$$

Since $\|A\|<1, \sum_{j=0}^{\infty}\|A\|^{j}$ will uniformly converge to $\frac{1}{1-\|A\|}$ Bromwich [2010]. If we let $x(0)=0$ and there exists a constant $0<L_{u}<\infty$, such that for all $k \geq 0$, $\|u(k)\| \leq L_{u}$, then

$$
\begin{aligned}
\|y(k+1)\| & \leq\|C\|\left(\sum_{j=0}^{\infty}\|A\|^{j}\right)\|B\| L_{u} \\
& =\frac{\|C\|\|B\|}{1-\|A\|} L_{u} .
\end{aligned}
$$

Let $L_{y}=\frac{\|C\|\|B\|}{1-\|A\|} L_{u}$, which is also a constant. For given $A, B, C$, and $L_{u}<\infty$, we will have $L_{y}<\infty$. By Definition 2 , system (1) is BIBO stable.

Then, by Lemma 2, system (1) is also BIBS stable.

From the above theorems, we can see that the asymptotic stability of the equilibrium point, the BIBS stability, and the BIBO stability of system (1) are related and can be verified by some tests and measurements. To check whether the condition given in Theorem 4 is satisfied, we first set 
$n$ linearly independent initial states, measure and record the following states, and then do some simple calculations. This data-based method requires the system state to be completely observable, which is a high demand that is not always satisfied. But, Theorem 4 has an advantage that we do not need to measure and record a large amount of output data as we do in Theorems 2 and 3. Besides, Theorem 4 can verify the BIBS stability and the asymptotic stability of the equilibrium point, while Theorem 2 cannot, which shows another advantage of Theorem 4 .

Lemma 5. Huang [2003] Assume that system (1) is completely state controllable. Then, the system is BIBS stable if and only if the equilibrium point $x=0$ is asymptotically stable.

Corollary 2. Suppose $q=n$. For system (1), if

1) $\operatorname{Rank}\{V\}=n$, where $V$ is defined in (3);

2) $\left\|y^{[j]}(\infty)\right\|=M_{j}<\infty(1 \leq j \leq m)$, where measured data $y^{[j]}(1), y^{[j]}(2), \ldots, y^{[j]}(n), \ldots$ are introduced in Subsection 2.1;

3) under no input condition, the initial states of system (1) can be set as in (9) and $\operatorname{rank}\{Q\}=n$, where $Q$ is defined in (10);

then the equilibrium point $x=0$ is asymptotically stable.

Proof. Since $q=n$, then by Condition 1) and Corollary 1 , system (1) is completely state controllable. According to Conditions 2), 3), and Theorem 3, system (1) is BIBS stable. From Lemma 5 , the equilibrium point $x=0$ is asymptotically stable.

From the above discussions, we can see that our databased methods can analyze the system characteristics only by using measured state and output data, and by doing some simple data arrangements. They neither identify the unknown matrices $A, B$, and $C$, nor calculate $C A^{l} B$ or $C A^{l}(0 \leq l \leq n-1)$. Therefore, our methods can avoid the corresponding identification errors and have lower computational complexity than traditional model-based system analysis methods.

\section{NUMERICAL EXAMPLE}

In this section, we use the following system as an example to illustrate our data-based system analysis methods.

$$
\left\{\begin{array}{l}
x(k+1)=\left[\begin{array}{rrr}
0.4 & 0.5 & -0.3 \\
0.3 & -0.2 & 0.1 \\
0.5 & -0.2 & 0.6
\end{array}\right] x(k)+\left[\begin{array}{rr}
0.7 & 1 \\
-0.4 & 0 \\
0.5 & -1
\end{array}\right] u(k), \\
y(k)=[-1,0,0.5] x(k) \quad(k \geq 0)
\end{array}\right.
$$

Here, $n=3, m=2$, and $q=1$. Set $u^{[1]}(k) \equiv[1,0]^{T}$,

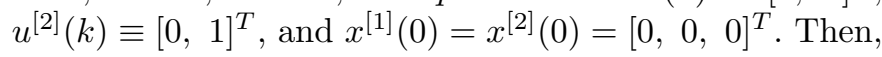

\begin{tabular}{|c|c|c|c|c|}
\hline \multicolumn{5}{|c|}{ Values of $y^{[1]}(k)$ and $y^{[2]}(k)(0 \leq k \leq n)$} \\
\hline Items & $k=0$ & $k=1$ & $k=2$ & $k=3$ \\
\hline$y^{[1]}(k)$ & 0 & -0.45 & -0.015 & 0.2295 \\
\hline$y^{[2]}(k)$ & 0 & -1.5 & -2.25 & -2.535 \\
\hline
\end{tabular}

As a result,

$$
V=[-0.45,-1.5,0.435,-0.75,0.2445,-0.285] \text {, }
$$

and $\operatorname{Rank}\{V\}=1=q$. By Theorem 1, system (17) is completely output controllable. This conclusion accords with the real case, since $\operatorname{Rank}\left\{W_{O C}\right\}=1=q$.

We then use Matlab programs to simulate the output and the state trajectories of system (17), which are shown below. In Fig. 1, the output trajectories starting from $y^{[1]}(0)=y^{[2]}(0)=0$, converge to two constants 0.569 and -2.1883 , respectively. Then, the condition of Theorem 2 and Condition 1) of Theorem 3 are both satisfied, and the system is BIBO stable. This can be confirmed by another two output trajectories, which start from $y^{[3]}(0)=-1.5$ and $y^{[4]}(0)=0.55$. The corresponding inputs are $u^{[3]}(k)=$ $\left[e^{(-0.2 k)}, 0.3\right]^{T}$ and $u^{[4]}(k)=[\sin (0.1 k \pi), \quad-\cos (0.2 k \pi)]^{T}$ $(k \geq 0)$, respectively, which are both bounded control inputs. We can see that the trajectories of $y^{[3]}(k)$ and $y^{[4]}(k)$ are both bounded.

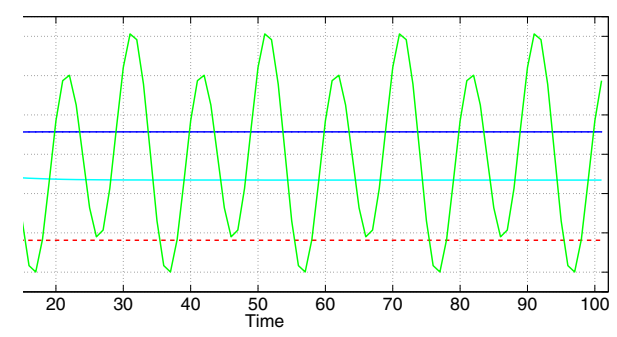

Fig. 1. Output trajectories of system (17)

Next, we set $\bar{x}^{[1]}(0)=\left[\begin{array}{lll}1, & 0,0\end{array}\right]^{T}, \bar{x}^{[2]}(0)=\left[\begin{array}{lll}0, & 1, & 0\end{array}\right]^{T}$,

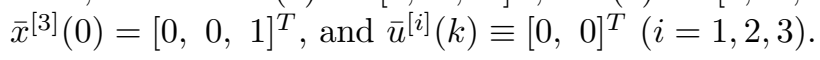

\begin{tabular}{|c|c|c|c|}
\hline \multicolumn{3}{|c|}{ Values of $\bar{y}^{[1]}(k), \bar{y}^{[2]}(k)$, and $\bar{y}^{[3]}(k)(0 \leq k \leq n-1)$} \\
\hline Items & $k=0$ & $k=1$ & $k=2$ \\
\hline $\bar{y}^{[1]}(k)$ & -1 & -0.15 & 0.06 \\
\hline $\bar{y}^{[2]}(k)$ & 0 & -0.6 & -0.075 \\
\hline $\bar{y}^{[3]}(k)$ & 0.5 & 0.6 & 0.345 \\
\hline
\end{tabular}

As a result, $Q=\left[\begin{array}{ccc}-1 & 0 & 0.5 \\ -0.15 & -0.6 & 0.6 \\ 0.06 & -0.075 & 0.345\end{array}\right]$, and $\operatorname{Rank}\{Q\}=$ $3=n$. Thus, Condition 2) of Theorem 3 is satisfied. By Theorem 3, system (17) is BIBS stable. By Lemma 3, the system is completely state observable. Such that we can measure the values of $\bar{x}^{[1]}(1), \bar{x}^{[2]}(1)$, and $\bar{x}^{[3]}(1)$.

\begin{tabular}{|c|c|c|}
\hline \multicolumn{3}{|c|}{ Values of $\bar{x}^{[1]}(1), \bar{x}^{[2]}(1)$, and $\bar{x}^{[3]}(1)$} \\
\hline $\bar{x}^{[1]}(1)$ & $\bar{x}^{[2]}(1)$ & $\bar{x}^{[3]}(1)$ \\
\hline$[0.4,0.3,0.5]^{T}$ & {$[0.5,-0.2,-0.2]^{T}$} & {$[-0.3,0.1,0.6]^{T}$} \\
\hline
\end{tabular}

Then,

$$
\begin{aligned}
& \left\|\left[\bar{x}^{[1]}(1), \bar{x}^{[2]}(1), \bar{x}^{[3]}(1)\right]\left[\bar{x}^{[1]}(0), \bar{x}^{[2]}(0), \bar{x}^{[3]}(0)\right]^{-1}\right\| \\
& =0.8799<1 .
\end{aligned}
$$

Therefore, the condition of Theorem 4 is satisfied, and the equilibrium point $x=0$ is asymptotically stable. This conclusion can be confirmed by one state trajectory starting from $[1,1,1]^{T}$ with $u(k) \equiv[0,0]^{T}$ shown in Fig. 2 . We can see that this trajectory converges to the origin as stated in Theorem 4 .

Moreover, the other four state trajectories start from $x^{[1]}(0)=x^{[2]}(0)=\left[\begin{array}{lll}0, & 0, & 0\end{array}\right]^{T}, x^{[3]}(0)=\left[\begin{array}{lll}0.5, & 1, & -2\end{array}\right]^{T}$, 
and $x^{[4]}(0)=[0,-0.6,1.1]^{T}$, whose inputs are $u^{[1]}(k) \equiv$ $[1,0]^{T}, u^{[2]}(k) \equiv[0,1]^{T}, u^{[3]}(k)=\left[e^{(-0.2 k)}, 0.3\right]^{T}$, and $u^{[4]}(k)=[\sin (0.1 k \pi),-\cos (0.2 k \pi)]^{T}(k \geq 0)$, respectively. As shown in Fig. 2, these state trajectories are all bounded, as stated in Theorem 3 .

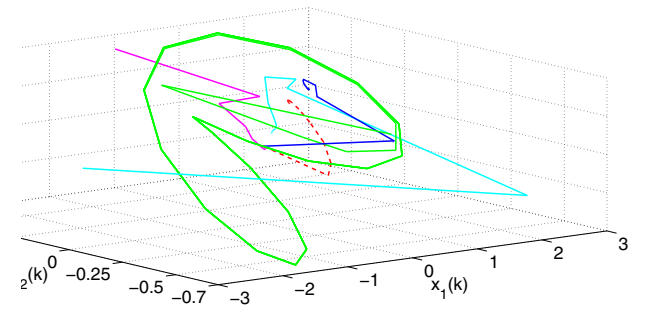

Fig. 2. State trajectories of system (17)

\section{CONCLUSIONS}

In this paper, we developed some data-based methods to analyze the characteristics of linear discrete-time systems. These data-based methods present a new perspective on system characteristics analysis.

Among our data-based analysis methods, Theorem 1 and Corollary 1 study the output controllability and the state controllability of the system, respectively, while Corollary 1 requires the system to have a special form. Theorem 2 concerns the BIBO stability, while Theorems 3 and 4 not only study the BIBO stability, but also study the BIBS stability and the stability of the equilibrium point. Then, Corollary 2 discusses a special case of Theorem 3 .

Unlike the traditional model-based methods, which need to perform system identification, our methods only utilize measured state and output data to directly verify the system properties without identifying the unknown parameter matrices. They thus have the advantage of lower computational complexity and can avoid the corresponding identification errors. But, the assumption that we can set the value of the initial state, actually requires the complete state controllability, which is a little high.

However, letting a system run in open loop for a long period of time to check its stabilities will be dangerous sometimes. Thus, at this stage, our work remains more academic than practical.

In the future, we hope to continue the study of the databased analysis methods for linear/nonlinear discrete-time systems, which do not need the priori assumptions and are more practical in application.

\section{REFERENCES}

P.J. Antsaklis and A.N. Michel. Linear Systems. Birkhäuser, Berlin, 2006.

G. Battistelli, A. Benavoli, and L. Chisci. Data-driven communication for state estimation with sensor networks. Automatica, 48(5): 926-935, 2012.

T.J.I. Bromwich. An Introduction to the Theory of Infinite Series. Nabu Press, 2010.
T. Chai, Z. Hou, F.L. Lewis, A. Hussain, and D. Zhao. Guest editorial: data-based control, modeling, and optimization. IEEE Trans. Neural Netw., 22(12): 21502153, 2011.

J. Ding, F. Ding, X.P. Liu, and G. Liu. Hierarchical least squares identification for linear SISO systems with dual-rate sampled-data. IEEE Trans. Autom. Control, 56(11): 2677-2683, 2011.

H. Hjalmarsson, J. Mårtensson, C.R. Rojas, and T. Söderström. On the accuracy in errors-in-variables identification compared to prediction-error identification. Automatica, 47(12): 2704-2712, 2011.

L. Huang. The Theoretical Basis of the Stability and Robustness. Science Press, Beijing, 2003.

B. Jayawardhana, H. Logemann, and E.P. Ryan. The circle criterion and input-to-state stability. IEEE Trans. Control Syst. Technol, 31(4): 32-67, Aug. 2011.

Z. Liu, S. Lin, and M.T. Tan. Sparse support vector machines with $L_{p}$ penalty for biomarker identification. IEEE/ACM Trans. Computational Biology and Bioinformatics, 7(1): 100-107, 2010.

C.H. Lu. Wavelet fuzzy neural networks for identification and predictive control of dynamic systems. IEEE Trans. Ind. Electron, 58(7): 3046-3058, 2011.

J. Michalkiewicz. Modified Kolmogorov's neural network in the identification of Hammerstein and Wiener systems. IEEE Trans. Neural Netw. Learn. Syst., 23(4): 657-662, 2012.

K.P. Mohandas. Modern Control Engineering. Sanguine Technical Publishers, Bangalore, 2006.

E.G. Moran and D.A.M. Labastida. Input-output stability for differential neural networks. 2011 IEEE Electronics, Robotics and Automotive Mechanics Conference (CERMA), Cuernavaca, Mexico, 53-58, Nov. 2011.

P. Rapisarda and H.L. Trentelman. Identification and data-driven model reduction of state-space representations of lossless and dissipative systems from noise-free data. Automatica, 47(8): 1721-1728, 2011.

A. Rensfelt and T. Söderström. Parametric identification of complex modulus. Automatica, 47(4): 813-818, 2011.

F.-L. Sun, Z.-H. Guan, X.-H. Zhang, and J.-C. Chen. Exponential-weighted input-to-state stability of hybrid impulsive switched systems. IET Control Theory \& Applications, 6(3): 430-436, Feb. 2012.

M. Verhaegen and V. Verdult. Filtering and System Identification: A Least Squares Approach. Cambridge University Press, New York, 2007.

Z. Wang and D. Liu. Data-based controllability and observability analysis of linear discrete-time systems. IEEE Trans. Neural Netw., 22(12): 2388-2392, Dec. 2011.

L. Zhang, Y.G. Xi, and W.D. Zhou. Identification and control of discrete-time nonlinear systems using affine support vector machines. International Journal of Artificial Intelligence Tools, 18(6): 929-947, 2009. 